\title{
Plasma Beta-Endorphin Levels Before and After Relief of Cancer Pain
}

Nabil El-Sheikh, MD, and Mark V. Boswell, MD, PhD

Background: Plasma $\beta$ endorphin (BE) is an endogenous peptide opioid derived form pro-opiomelanocortin. Although the role of plasma $B E$ in pain regulation is unclear, plasma BE levels have been reported to correlate inversely with pain levels in cancer pain.

Objectives: To measure BE levels in patients with cancer before and after pain relief with different analgesic modalities, to evaluate the relationship between cancer pain, pain control and plasma BE levels.

Methods: Prospective interventional study conducted in a university hospital setting. Patients with intractable pain due to upper abdominal visceral malignancies (pancreatic, liver and gastric cancers) agreed to participate in the study. Pain relief was provided with one of four primary

Beta-endorphin (BE), an endogenous peptide opioid derived form proopiomelanocortin, is a neurohormone secreted by the anterior pituitary into the systemic circulation. Endorphins are found in regions of the brain involved in the perception of pain, including the nucleus accumbens and the arcuate nucleus.

Although the role of plasma $\mathrm{BE}$ in pain regulation is unclear, plasma $\mathrm{BE}$ levels have been reported to correlate inversely with pain levels in cancer pain $(1,2)$ chronic daily headache $(3)$ and postoperative pain (4). That is, plasma BE levels are lower in patients with poorly controlled pain, and increase with pain relief.

In this study, we measured plasma

From The Department of Anesthesiology, University of Tanta, Tanta, Egypt and Department of Anesthesiology, School of Medicine, Case Western Reserve University and University Hospitals of Cleveland, Cleveland, Ohio. Address Correspondence: Mark V. Boswell, MD, PhD, University Hospitals, 11100 Euclid Avenue, Cleveland, Ohio 44106

Email: mark.boswell@uhhs.com

Funding: There was no external funding in prepara-

tion of this manuscript.

Conflict of Interest: None modalities: 1) oral continuous release morphine, 2) thoracic epidural morphine boluses, 3) alcohol celiac plexus blocks or 4) interpleural infusions of bupivacaine. Blood samples for plasma BE levels were taken before treatment and at the time of maximal pain relief obtained with the particular treatment modality. Pain levels were determined using a $10 \mathrm{~cm}$ visual analogue scale. BE levels were measured by competitive radioimmunoassay using ${ }^{125} \mid \beta$ endorphin.

Results: Average pain scores decreased from $7.3+1.27(\mathrm{SD})$ before treatment to 1.2 $+1.18(\mathrm{SD})$ after treatment $(\mathrm{p}<0.0001)$. Satisfactory pain relief was obtained with each of the four treatment modalities and was associated with a significant increase in plasma BE levels. The mean plasma BE level for all groups before treatment was $18.9 \pm 5.4 \mathrm{pg} /$

BE levels in patients suffering from intractable pain due to upper abdominal malignancies, before and after pain relief, to evaluate the relationship between cancer pain, pain control and plasma BE levels. BE levels were determined by competitive radioimmunoassay using ${ }^{125} \mathrm{I} \beta$ endorphin.

\section{Methods}

After institutional review board approval and informed consent, 20 patients with intractable upper abdominal cancer pain participated in the study. Tumor diagnoses included pancreatic, liver and gastric cancers.

Pain relief was achieved with oral controlled release morphine (MS Contin (), thoracic epidural morphine injections, alcohol celiac plexus blocks or interpleural bupivacaine infusions. For the oral morphine group, controlled release morphine tablets were administered every 12 hours. Additional doses of $10 \mathrm{mg}$ of immediate release morphine were given every 4 hours as needed for breakthrough pain. Thoracic epidural catheters were in- $\mathrm{ml}$ (range 2.0 to $29.6 \mathrm{pg} / \mathrm{ml}$ ) compared to $38.7 \pm 17.6 \mathrm{pg} / \mathrm{ml}$ (range 13.2 to $67.9 \mathrm{pg} / \mathrm{ml}$ ) after pain relief $(\mathrm{p}<0.0001)$. Plasma BE levels increased with improved pain control with each of the four analgesic modalities, including oral and epidural morphine.

Conclusions: Plasma BE levels increased with improved pain control in patients with upper abdominal gastrointestinal malignancies. Although the role of plasma BE in pain pathophysiology is unclear, it appears that pain relief per se, and not the analgesic technique, modulates plasma BE levels. This suggests that plasma BE levels may serve as an objective measure of cancer pain severity and corroborate the patient's report of pain relief.

Keywords: Plasma endorphins, morphine, malignancy, celiac plexus, epidural analgesia.

serted between $\mathrm{T} 5$ and $\mathrm{T} 12$, depending on the level of the most painful dermatome. Epidural morphine was administered in doses of 0.03 to $0.05 \mathrm{mg} / \mathrm{kg}$ in $10 \mathrm{ml}$ of saline as intermittent injections, with a minimum dose interval of 4 to 6 hours. Celiac plexus blocks were done with CT guidance, using bilateral needle placement. After a test dose of $0.25 \%$ bupivacaine, 15 $\mathrm{ml}$ of $100 \%$ alcohol was injected bilaterally. Immediate release oral morphine was provided for breakthrough pain as needed to supplement pain relief provided by the celiac block. Unilateral interpleural catheters were inserted on the most painful side, between the seventh and eighth ribs, 8 to $10 \mathrm{~cm}$ lateral to midline, and $0.5 \%$ bupivacaine was infused for pain relief. Breakthrough pain was treated with immediate release morphine.

Blood samples for BE levels were taken before treatment and at the time of maximal pain relief obtained with the particular treatment modality. Pain levels were determined using a $10 \mathrm{~cm}$ visual analogue scale ("no pain" - $0 \mathrm{~cm}$ and "the worst pain possible" - 
Table 1. Patient characteristics, type of pain treatment and pain scores before and after treatment.

\begin{tabular}{|l|c|c|c|}
\hline Type of Analgesia & Number of Patients & $\begin{array}{c}\text { Pain Scores* Before } \\
\text { Treatment }\end{array}$ & $\begin{array}{c}\text { Pain Scores* After } \\
\text { Treatment }\end{array}$ \\
\hline Epidural Morphine & 6 & $6.7 \pm 1.03$ & $1.0 \pm 1.26$ \\
\hline MS Contin & 3 & $7.3 \pm 1.53$ & $3.0 \pm 0.00$ \\
\hline Celiac Plexus & 3 & $8.7 \pm 1.15$ & $1.7 \pm 0.58$ \\
\hline Interpleural & 8 & $7.1 \pm 1.46$ & $0.4 \pm 0.52$ \\
\hline Average scores before and after treatment: & $7.3 \pm 1.27$ & $1.2 \pm 1.18$ \\
\hline
\end{tabular}

* Mean \pm SD

Before and after treatment pain scores for each treatment group were significant $(P<0.01)$. With Bonferroni corrections, the differences in pain scores between treatment groups were not statistically significant.

$10 \mathrm{~cm})$. All endorphin samples were drawn at 9 AM to avoid diurnal variation. Plasma samples were purified on C-18 Sep Pak cartridges and stored frozen at $-20^{\circ} \mathrm{C}$ until analyzed by competitive radioimmunoassay, using ${ }^{125} \mathrm{I} \beta$ endorphin.

Statistical significance was determined with a two-tailed Student's t-test with Bonferroni/Dunn corrections for multiple comparisons. Regression analysis of endorphin levels was done with a statistical computer package (StatView, Abacus Concepts, Berkeley, CA).

\section{ResULTS}

Average patient age was 58 years and mean weight was $70 \mathrm{~kg}$. Patient treatment categories and average pain scores before and after treatment are shown in Table 1. Average pain scores decreased from $7.3 \pm$
1.27 (SD) before treatment to $1.2 \pm 1.18$ $(S D)$ with pain relief $(p<0.0001)$. Satisfactory pain relief was obtained with each of the four treatment modalities. The pre-treatment pain scores for each treatment group were not significantly different from each other. Similarly, posttreatment pain scores were not statistically different between the four modalities. Therefore, the data for pre and post treatment endorphin levels, respectively, were pooled.

Satisfactory analgesia was associated with a significant increase in plasma $\mathrm{BE}$ levels. The mean plasma BE level before treatment was $18.9 \pm 5.4 \mathrm{pg} / \mathrm{ml}$ (range 2.0 to $29.6 \mathrm{pg} / \mathrm{ml}$ ) compared to $38.7 \pm 17.6 \mathrm{pg} /$ $\mathrm{ml}$ (range 13.2 to $67.9 \mathrm{pg} / \mathrm{ml}$ ) after pain relief ( $\mathrm{p}<0.0001)$ (Fig. 1).

Although samples sizes were small, it does not appear that plasma BE levels were significantly different between spe-
Table 2. Daily morphin requirements for each analgesic modality

\begin{tabular}{|c|c|}
\hline Analgesic Modality & $\begin{array}{c}\text { Average } \\
\text { Daily Opioid } \\
\text { Requirement }\end{array}$ \\
\hline Epidural morphine & $11 \mathrm{mg}$ (epidurally) \\
\hline Oral morphine & $76 \mathrm{mg}$ \\
\hline Celiac plexus block & $6 \mathrm{mg}$ \\
\hline Interpleural infusion & $8 \mathrm{mg}$ \\
\hline
\end{tabular}

Satisfactory pain relief was obtained with each of the treatment modalities, despite the wide variation in total opioid doses required.

cific pain treatment modalities (Fig. 2). For comparison, the average oral morphine requirements for the individual treatment groups are shown in Table 2. Thus, morphine doses and route of administration (oral vs. epidural) did not appear to correlate with plasma BE levels. Rather, plasma BE levels correlated with the level of pain relief. Regression analysis of the data is depicted in Fig. 3.

\section{Discussion}

Plasma BE levels were increased following relief of cancer pain compared to baseline pain scores prior to pain relief. This study is in agreement with the reports by Lopez and colleagues (1) and Mystakidou et al (2), who found lower plasma BE levels in patients with poorly controlled pain due to breast, lung and visceral malignancies, and increased plasma BE levels after pain reduction. Both

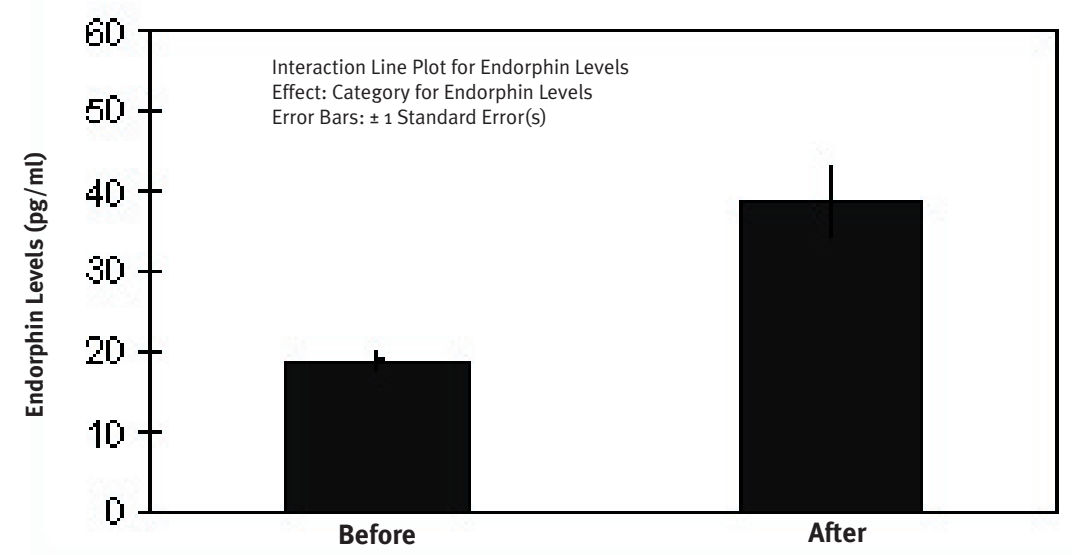

Average plasma endorphin levels for patients before treatment and after treatment (with maximum pain relief) are shown. The difference in plasma BE levels before and after treatment was statistically significant $(\mathrm{p}<.0001)$. Differences between specific modalities before and after treatment, respectively, were not significant and data from the treatment groups were pooled.

Fig 1. Endorphin levels before and after pain relief. 


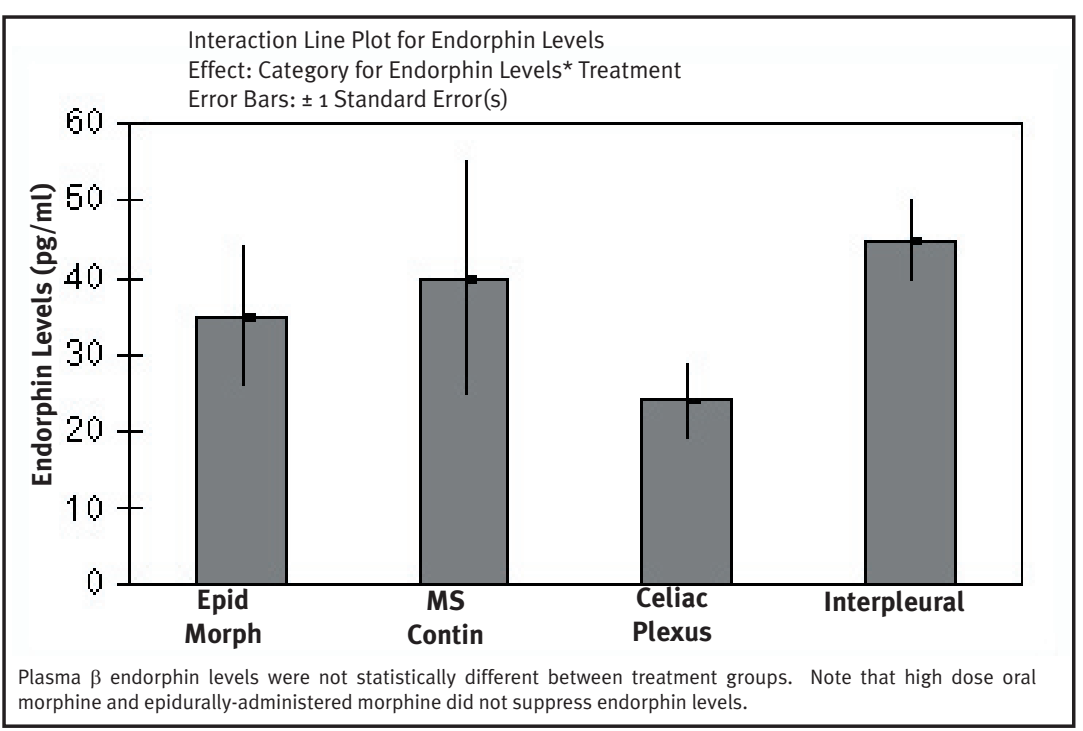

Fig 2. Endorphin levels and treatment modalities.

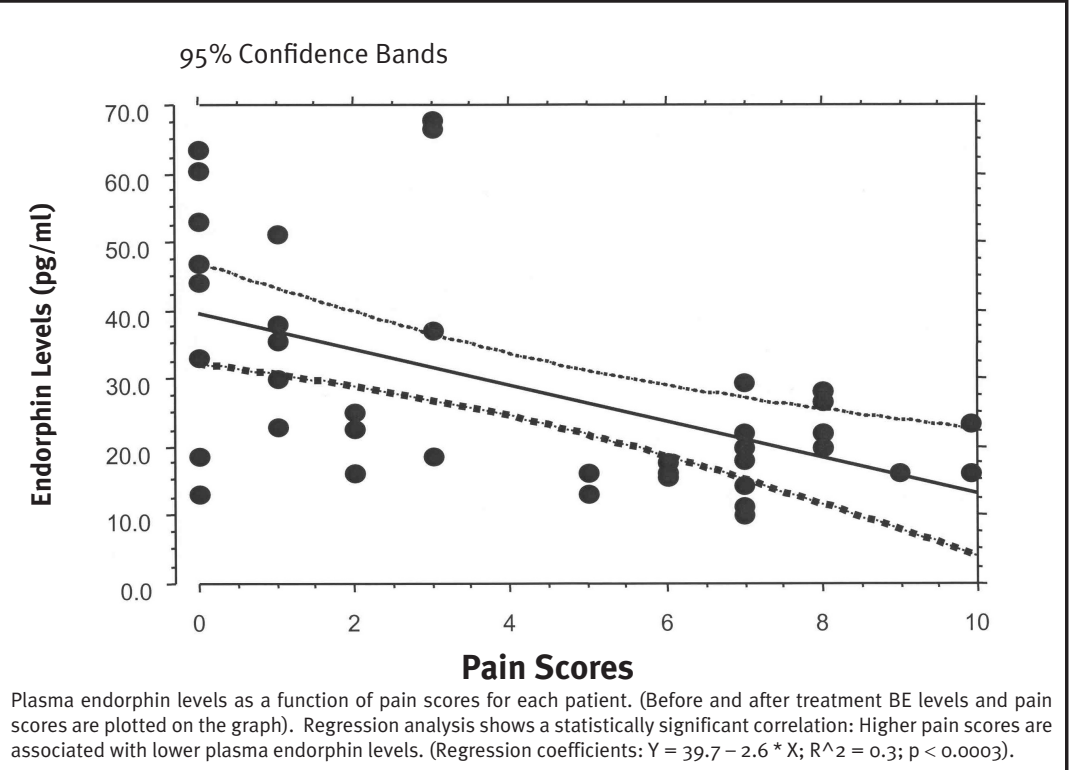

Fig 3. Endorphin levels and pain scores.

of those studies used analgesic techniques that did not involve opioids. Similarly, Befon and colleagues (5) found that treatment with subcutaneous octreotide, a somatostatin analogue, improved pain due to gastrointestinal cancers and resulted in increases levels of plasma BE.

In our study, patients received oral or parenteral opioids, either as the primary or supplemental analgesic. Although the role of plasma BE in pain pathophysiology is unclear, it appears that pain relief per $s e$, and not the analgesic technique, mod- pain control remains unclear, although it is assumed that plasma endorphin levels correlate with CSF levels. Opioid receptors are pharmacologically classified as mu, delta, kappa and epsilon. The epsilon opioid receptor system appears to be stimulated supraspinally by the endogenous opioid peptide $\beta$ endorphin (6). Stimulation of the epsilon receptor induces the release of met-enkephalin, which then acts on spinal delta opioid receptors to produce analgesia. The epsilon receptor-mediated descending system appears to be distinct from the mu opioid receptor system. This suggests that the endorphin system is a parallel analgesic system, and in some manner plasma endorphin levels reflect the current levels of pain or analgesia.

\section{CONCLUSION}

In summary, this study confirms that plasma BE levels increase with improved pain control in upper abdominal gastrointestinal malignancy. This suggests that plasma BE levels may serve as an objective measure of cancer pain severity and corroborate the patient's report of pain relief.

Author Affiliation:
Nabil El-Sheikh, MD
Department of Anesthesiology
University of Tanta
Tanta, Egypt
Mark V. Boswell, MD, PhD
Associate Professor of Anesthesiology
Department of Anesthesiology
School of Medicine
Case Western Reserve University and
University Hospitals of Cleveland
Cleveland, Ohio.
Email: mark.boswell@uhhs.com

\section{REFERENCES}

ulates plasma BE levels. This is evident because pain relief from parenteral opioids, epidurally-administered opioids and local anesthetic infusions correlated with increased plasma BE levels. Moreover, contrary to conventional wisdom, analgesic techniques that used oral or epidural morphine did not suppress BE levels. Intuitively, one might have predicted a fall in endogenous endorphins with administration of exogenous opioids, which was not the case in the present study.

The relevance of $\beta$ endorphins in
1. Lopez JA, Peran F, Altuzarra A et al. Plasmatic B-endorphin levels and thalamic surgery for pain. Neurosurgical Res 1985; 7:35-38.

2. Mystakidou $\mathrm{K}$, Befon $\mathrm{S}$, Hondros $\mathrm{K}$ et al. Continuous subcutaneous administration of high-dose salmon calcitonin in bone metastases: pain control and beta-endorphin plasma levels. J Pain Symptom Manage 1999; 18:323-330.

3. Facchinetti F, Nappi G, Savoldi F, Genazzani AR. Primary headaches: reduced circulating B-lipotropin and B-endorphin levels with impaired reactivity to acupuncture. Cephalalgia 1981; 1:195-201. 
4. Leonard TM, Klem SA, Asher MA et al. Relationship between pain severity and serum B-endorphin levels in postoperative patients. Pharmacotherapy 1993; 13:378-381.
Befon S, Mystakidou K, Lyra M et al. Con- 6. tinuous subcutaneous octreotide in gastrointestinal cancer patients: control and beta-endorphin levels. Anticancer Res 2000; 20:4039-4046.
6. Tseng LF. Evidence for epsilon-opioid receptor-mediated beta-endorphin-induced analgesia. Trends Pharmacol Sci 2001; 22:623-630. 